\title{
THE TITCHMARSH SEMI-GROUP
}

\section{G. L. KRABBE}

Introduction. Let $S$ be the set of all complex-valued functions defined on $\Omega=\{0, \pm 1, \pm 2, \cdots\}$. We suppose $p>1$, and consider the space $S_{p}$ of all members $a$ of $S$ such that $\|a\|_{p}=\left(\sum_{\nu=-\infty}^{\infty}\left|a_{p}\right|^{p}\right)^{1 / p}<\infty$. The letters $\alpha$ and $\lambda$ will henceforth denote complex numbers.

The transformation $T_{\alpha}$ is defined as follows: if $a \in S_{p}$, then $T_{\alpha} a$ is the member $c$ of $S$ satisfying

$$
\left[T_{\alpha} a\right]_{n}=c_{n}=\sum_{\nu=-\infty}^{\infty}(-1)^{n+\nu} \frac{\sin \alpha \pi}{(n+\alpha-\nu) \pi} a_{\nu} \quad(n \in \Omega) .
$$

For any $a$ in $S_{p}$, we define $G a$ to be the member $x$ of $S$ such that

$$
[G a]_{n}=x_{n}=\sum_{\nu=-\infty}^{\infty}(-1)^{n+\nu} \frac{1}{n-\nu} a_{\nu}, \quad \nu \neq n(n \in \Omega) .
$$

M. Riesz [10] has shown that both $T_{\alpha}$ and $G$ are bounded operators. An operator essentially identical to $T_{\alpha}$ was studied by E. C. Titchmarsh $[12 ; 13]$ in the case $\alpha=1 / 2$.

In this paper we prove that:

(i) $T_{\alpha}$ is an entire function.

(ii) $T_{0} a=a$ and $T_{\alpha} T_{\lambda} a=T_{\alpha+\lambda} a$ for any $a$ in $S_{p}$.

(iii) The operator $G$ is the infinitesimal generator of what we call the Titchmarsh semi-group $\left\{T_{\alpha} \mid \alpha\right\}$. Moreover $T_{\alpha}=\exp \alpha G$ for all complex $\alpha$.

These results are derived from the following property $\left(\mathrm{A}_{p}\right)$.

$\left(\mathrm{A}_{p}\right)$ There is a nondecreasing function $f$ such that $\left\|T_{\alpha}\right\|_{p} \leqq f(|\operatorname{Im} \alpha|)$.

If $\operatorname{Im} \alpha=0$, it is readily inferred from (ii) and $\left(\mathrm{A}_{p}\right)$ that $T_{\alpha}$ is weakly-almost-periodic in the sense of Lorch [6]; its spectrum $\sigma\left(T_{\alpha}\right)$ is therefore on the circumference of the unit-circle. ${ }^{1}$

Our basic lemmas depend heavily upon the methods developed in [12]. W. Ferrar [1] has verified a special case of (ii). The operator $T_{\alpha}$ forms the basis of an article by $\mathrm{H}$. Hadwiger [2]; in E. Hille's review [4] of [2] is found the remark that (i) and (ii) hold when $p=2$. A corresponding generalization of $[2]$ is made possible by our removal of this restriction. Among various applications of $T_{\alpha}$ we mention: inter-

Presented to the Society, June 19, 1954; received by the editors April 23, 1953 and, in revised form, May 10, 1954 and July 1, 1954.

1 This follows from $[6$, p. 36]. We intend to show in a later article that $\sigma(i G)$ $=[-\pi, \pi]$ and use these properties to illustrate a general theorem based on [6]. 
polation theory [1], fractional differentiation [2], and the derivation by Titchmarsh of the fundamental properties of the Hilbert transformation [12]. An extensive literature [3] deals with the operators obtained from (1) and (2) by imposing the conditions $n>0, \nu>0$. Some of the connections of this subject with problems in other fields are mentioned in $[8 ; 7]$.

1. Preliminaries. As usual, $\left\|T_{\alpha}\right\|_{p}=\sup \left\|T_{\alpha} a\right\|_{p} ;\|a\|_{p}=1, a \in S$. We shall consistently write $p^{\prime}=p /(p-1)$. Let $t^{\alpha}$ be the member of $S$ such that $t_{n}^{\alpha}$ is the $n$th Fourier coefficient of $\exp (-i \theta \alpha)$. Thus

$$
t_{n}^{\alpha}=(-1)^{n} \xi_{\alpha} /(n+\alpha) \quad \text { if } \quad \xi_{\alpha}=(\sin \alpha \pi) / \pi \quad \text { and } n \neq-\alpha, n \in \Omega ;
$$

moreover $t_{-\alpha}^{\alpha}=1$ if $\alpha \in \Omega$. In case $a \in S_{p}$ and $b \in S_{p^{\prime}}$, then $b * a$ denotes the member $x$ of $S$ such that

$$
[b * a]_{n}=x_{n}=\sum_{\nu=-\infty}^{\infty} b_{v} a_{n-\nu} \quad \text { when } n \in \Omega .
$$

Consequently $T_{\alpha} a=t^{\alpha} * a$. We shall show in $\S 5$ that $G a=d * a$, where $d_{n}$ is the derivative of $t_{n}^{\alpha}$ at $\alpha=0$. If $m \in \Omega$ then [ $\left.T_{m} a\right]_{n}=a_{m+n}$, so that

$$
T_{0} a=a \text { and }\left\|T_{m}\right\|_{p}=1 \quad \text { when } m \in \Omega .
$$

The Parseval and Fischer-Riesz theorems yield immediately (see $[10 ; 14])$

$$
\left\|T_{\alpha}\right\|_{2} \leqq \zeta(\alpha)=\exp (\pi|\operatorname{Im} \alpha|) \text { and }\left\|t^{\alpha}\right\|_{2} \leqq \zeta(\alpha) .
$$

1.1 Notation. From now on, we denote by $P_{p}$ the set of all members $c$ of $S$ such that $c_{n}=0$ for all $|n|$ sufficiently large, and satisfying $\|c\|_{p}=1$.

We say that $\phi(\alpha) \in \mathcal{F}$ if $\phi(\alpha) \in[0, \infty)$ and if moreover $\phi$ is a function such that

(a) $\phi(\alpha)$ is bounded when $\alpha \in[0,1]$,

(b) there exists a nondecreasing function $f$ mapping $[0, \infty)$ into itself, and satisfying $\phi(\alpha) \leqq f(|\operatorname{Im} \alpha|)$ whenever $\operatorname{Rl} \alpha=0$.

1.2 Remark. $^{2}$ If $\|x-y\|_{p} \leqq h_{1}(\alpha) \in \mathcal{F}$ and $\|x\|_{p} \leqq h_{2}(\alpha) \in \mathcal{F}$, then $\|y\|_{p} \leqq h_{3}(\alpha) \in \mathcal{F}$.

\subsection{Lemma. If $\left\|T_{\alpha}\right\|_{p}<\infty$, then (ii) holds.}

Proof. Suppose $a \in S_{p}$ and let $K$ be an arbitrary pair $\left(K_{1}, K_{2}\right)$ of members of $\Omega$. We denote by $\{a ; K\}$ the member $x$ of $S$ such that: $x_{n}=a_{n}$ if $K_{1}<n<K_{2}$, and $x_{n}=0$ otherwise $(n \in \Omega)$. Thus, if $n \in \Omega$,

${ }^{2}$ For convenience and brevity, the statement that there exists a function $h$ such that $\|z\|_{p} \leqq h(\alpha)$ and $h(\alpha) \in \mathcal{F}$ will be expressed symbolically by writing $\|z\|_{p} \leqq h(\alpha) \in \mathcal{F}$. 


$$
\left[T_{\alpha} a\right]_{n}=\lim \left[T_{\alpha}\{a ; K\}\right]_{n} \quad \text { as } K_{1} \rightarrow-\infty, K_{2} \rightarrow \infty .
$$

By Hölder's inequality

$$
\left|\left[T_{\alpha} c\right]_{n}\right|=\left|\left[t^{\alpha} * c\right]_{n}\right| \leqq\left\|t^{\alpha}\right\|_{p^{\prime}}\|c\|_{p}
$$

Hence, taking $c=T_{\lambda} a-T_{\lambda}\{a ; K\}=T_{\lambda}(a-\{a ; K\})$,

(6) $\left|\left[T_{\alpha}\left(T_{\lambda} a\right)\right]_{n}-\left[T_{\alpha}\left(T_{\lambda}\{a ; K\}\right)\right]_{n}\right| \leqq\left\|t^{\alpha}\right\|\left\|_{p^{\prime}}\right\| T_{\lambda}\left\|_{p}\right\| a-\{a ; K\} \|_{p}$.

It can easily be shown [10] that (ii) holds in the case $p=2$. But $\{a ; K\} \in S_{2}$, and we can therefore replace, in (6), $T_{\alpha} T_{\lambda}\{a ; K\}$ by $T_{\alpha+\lambda}\{a ; K\}$. The conclusion is now obtained by taking $\lim K_{1} \rightarrow-\infty$ and $\lim K_{2} \rightarrow \infty$ of both sides of (6), using (5) and the fact that

$$
\lim \|a-\{a ; K\}\|_{p}=\lim \sum_{n=-\infty}^{K_{1}}\left|a_{n}\right|^{p}+\lim \sum_{n=K_{2}}^{\infty}\left|a_{n}\right|^{p}=0 .
$$

2. Basic lemmas. In the present section, we prove that $\left(A_{p}\right)$ holds for any $p$ in $M=\left\{2,2^{2}, 2^{3}, \cdots\right\}$. We henceforth write $s^{\prime}=s /(s-1)$ and call $d$ the member of $S$ such that $d_{0}=0, d_{n}=(-1)^{n} / n$ when $n \neq 0$. If $a \in S$ and $b \in S$, it will be convenient to define $a b$ and $a^{2}$ by $[a b]_{n}=a_{n} b_{n}$ and $\left[a^{2}\right]_{n}=a_{n}^{2}$ respectively. Note that if $s>1$

$$
\|a b\|_{s} \leqq\|a\|_{2 s}\|b\|_{2 s} \text { and }\left\|a^{2}\right\|_{s}=\|a\|_{2 s}^{2} \text {. }
$$

2.1 Lemma. Suppose $s \geqq 2$ and $\tau=1,2$. Then

$$
\left\|\left\{\left(t^{\alpha}\right)^{\tau} d\right\} * x\right\|_{\mathrm{o}} \leqq \Psi_{\mathrm{*}}^{(\tau)}(\alpha) \in \mathcal{F} \quad \text { when } x \in \mathcal{P}_{\mathrm{s}} .
$$

Proof. If $z=\left\{\left(t^{\alpha}\right)^{\tau} d\right\} * x$ and $n \in \Omega$, then by Hölder's inequality

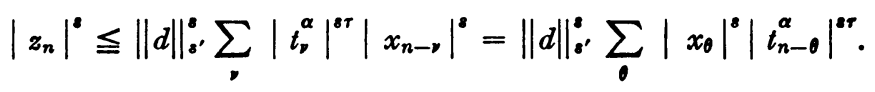

Now $t_{n-\theta}^{\alpha}=t_{n}^{\alpha-\theta}$. We therefore ${ }^{8}$ infer from (4) that

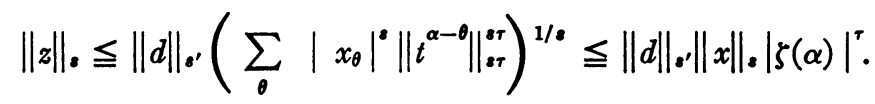

We conclude the proof by observing that $|\zeta(\alpha)|^{\tau} \in \mathcal{F}$.

2.2 Lemma. Suppose $s \geqq 2$ and $\tau=1,2$. Then

$$
\left\|\left[\left\{\left(t^{\alpha}\right)^{\tau-1} \xi_{\alpha} d\right\} * x\right]-\left[\left(t^{\alpha}\right)^{\tau} * x\right]\right\|_{.} \leqq \Phi_{s}^{(\tau)}(\alpha) \in \mathcal{F} \quad \text { when } x \in \mathcal{P}_{\text {s. }}
$$

Proof. Call $y=\left\{\left(t^{\alpha}\right)^{r-1} \xi_{\alpha} d\right\}-\left(t^{\alpha}\right)^{r}$. It is easily checked that

${ }^{3}$ Since $s \tau \geqq 2$, and thus $\left\|t^{\alpha-\theta}\right\|_{0 r} \leqq\left\|t^{\alpha-\theta}\right\|_{2} \leqq \zeta(\alpha)$. 


$$
y_{n}=(-1)^{n} \alpha\left(t_{n}^{\alpha}\right)^{\tau} \cdot d_{n}-\left(t_{0}^{\alpha}\right)^{\tau} \cdot t_{n}^{0}
$$

so that if $x \in \mathcal{P}_{s}$,

$$
\|y * x\|_{s} \leqq|\alpha| \cdot\left\|\left\{\left(t^{\alpha}\right)^{\tau} d\right\} * x\right\|_{s}+\left|t_{0}^{\alpha}\right|^{\tau} \cdot\left\|t^{0} * x\right\|_{8} .
$$

But $t^{0} * x=x,\left|t_{0}^{\alpha}\right|^{\tau} \in \mathcal{F}$, and by 2.1

$$
\|y * x\|_{s} \leqq|\alpha| \Psi_{s}^{(\tau)}(\alpha)+\left|t_{0}^{\alpha}\right|^{\tau}=\Phi_{s}^{(\tau)}(\alpha) \in \mathcal{F} .
$$

2.3 Lemma. If $r \geqq 2$ there exists a function $g_{r}$ with $g_{r}(\alpha) \in \mathcal{F}$ and such that for all $a$ in $\mathcal{P}_{2 r}$

$$
\left\|T_{\alpha} a\right\|_{2 r}^{2}-2 \cdot\left\|T_{\alpha}\right\|_{r}\left(\left\|T_{\alpha} a\right\|_{2 r}+g_{r}(\alpha)\right) \leqq f_{r}(\alpha) \in \mathcal{F} .
$$

Proof. Suppose $a \in \mathcal{P}_{2 r}$ and call $h=\left(T_{\alpha} a\right)^{2}-\left[\left(t^{\alpha}\right)^{2} * a^{2}\right]$. We first note that

$$
h_{n}=\sum_{\nu} \sum_{\theta \neq \nu} \underset{t_{\nu} t_{\theta}^{\alpha} a_{n-\nu} a_{n-\theta} .}{\alpha}
$$

By (7), $\left\|a^{2}\right\|_{r}=\|a\|_{2 r}^{2}=1$, so that $a^{2} \in \mathcal{P}_{r}$. We can therefore conclude from $2.2,2.1$, and 1.2 that $\left\|\left(t^{\alpha}\right)^{2} * a^{2}\right\|_{r} \leqq f_{r}(\alpha) \in \mathcal{F}$. From the definition of $h$ now follows that

$$
\left\|T_{\alpha} a\right\|_{2 r}^{2}-\|h\|_{r} \leqq f_{r}(\alpha) \in \mathcal{F} .
$$

Suppose $x \in S$; if we define $-x$ by $[-x]_{n}=(-1)^{n} x_{n}$, then $\|-x\|_{r}=\|x\|_{r}$ and $(-z) * x=-[z *(-x)]$. This enables us to derive from (7) that

$$
\left\|\left(-t^{\alpha}\right) *(a c)\right\|_{r}=\left\|T_{\alpha}(-a c)\right\|_{r} \leqq\left\|T_{\alpha}\right\|_{r}\|a c\|_{r} \leqq\left\|T_{\alpha}\right\|_{r}\|c\|_{2 r} .
$$

It is immediately verified that $t_{v}^{\alpha} t_{\theta}^{\alpha}=\xi_{\alpha}\left(\left[-t^{\alpha}\right]_{\nu} d_{m}+\left[-t^{\alpha}\right]_{\theta} d_{-m}\right)$ if $m=\theta-\nu \neq 0$ so that, by (8),

$$
h_{n}=2 \sum_{\nu}\left[-t^{\alpha}\right]_{\nu} a_{n-\nu} \sum_{m} \xi_{\alpha} d_{m} a_{(n-\nu)-m}=2\left[\left(-t^{\alpha}\right) *\left(a\left\{\xi_{\alpha} d * a\right\}\right)\right]_{n} .
$$

Using now (10) and 2.2,

$$
\|h\|_{r} \leqq 2\left\|T_{\alpha}\right\|_{r}\left\|\xi_{\alpha} d * a\right\|_{2 r} \leqq 2\left\|T_{\alpha}\right\|_{r}\left(\left\|t^{\alpha} * a\right\|_{2 r}+\Phi_{2 r}^{(1)}(\alpha)\right) .
$$

The conclusion follows from (9).

2.4 FinAL LEMMA. Whenever $\left\|T_{\alpha}\right\|_{r} \leqq \phi_{r}(\alpha) \in \mathcal{F}$ for some $r \geqq 2$, then $\left\|T_{\alpha}\right\|_{2 r} \leqq \phi_{2 r}(\alpha) \in \mathcal{F}$.

Proof. If $a \in \mathcal{P}_{2 r}$ and $x=T_{\alpha} a$, then by 2.3

$$
\|x\|_{2 r}^{2}-2 \phi_{r}(\alpha)\|x\|_{2 r} \leqq 2 \phi_{r}(\alpha) \cdot g_{r}(\alpha)+f_{r}(\alpha)=h_{1}(\alpha) \in \mathcal{F} .
$$


Adding $\left(\phi_{r}(\alpha)\right)^{2}$ to both sides of the inequality,

$$
\left\{\|x\|_{2 r}^{2}-\phi_{r}(\alpha)\right\}^{2} \leqq\left(\phi_{r}(\alpha)\right)^{2}+h_{1}(\alpha)=h_{2}(\alpha) \in \mathcal{F} .
$$

This yields the conclusion

$$
\left\|T_{\alpha} a\right\|_{2 r}=\|x\|_{2 r} \leqq \phi_{r}(\alpha)+\left(h_{2}(\alpha)\right)^{1 / 2}=\phi_{2 r}(\alpha) \in \mathcal{F} .
$$

2.5 Theorem. (As) holds for any $s$ in $M$.

Proof. From (4) and 2.4 we can conclude that for any $s$ in $M$, $\left\|T_{\lambda}\right\|_{s} \leqq \phi_{s}(\lambda) \in \mathcal{F}$. Set $\alpha=\alpha^{0}+\alpha^{\prime}+i \alpha^{\prime \prime}$, where $\alpha^{0} \in \Omega, \alpha^{\prime} \in[0,1]$, and $\alpha^{\prime \prime} \in(-\infty, \infty)$; then by 1.3 and (3),

$$
\left\|T_{\alpha}\right\|_{\delta}=\left\|T_{\alpha^{0}} T_{\alpha^{\prime}+i \alpha^{\prime \prime}}\right\|_{\delta} \leqq\left\|T_{\alpha^{\prime}+i \alpha^{\prime \prime}}\right\|_{\delta} \leqq \phi_{\delta}\left(\alpha^{\prime}\right)\left\|T_{i \alpha^{\prime \prime}}\right\|_{\text {.. }}
$$

Now $\phi_{s}(\lambda) \in \mathcal{F}, \alpha^{\prime} \in[0,1]$, and $\phi_{s}\left(\alpha^{\prime}\right)$ is therefore bounded by some number $k$. Moreover, $\left\|T_{i \alpha^{\prime \prime}}\right\|_{s} \leqq \phi_{8}\left(i \alpha^{\prime \prime}\right)$ and we infer from 1.1(b) and $\mathrm{Rl} i \alpha^{\prime \prime}=0$ that there exists a nondecreasing function $f$ such that $\phi_{s}\left(i \alpha^{\prime \prime}\right) \leqq f\left(\left|\alpha^{\prime \prime}\right|\right)$. Collecting results: $\left\|T_{\alpha}\right\|_{s} \leqq k \cdot f\left(\left|\alpha^{\prime \prime}\right|\right)$.

\section{The main theorems.}

3.1 LemMA. If $p>1$, there exists a member $s$ of $M$ such that $\left\|T_{\alpha}\right\|_{p}$ $\leqq\left\|T_{\alpha}\right\|_{8}$.

We have already indicated that $T_{\alpha}$ is essentially the operator studied by Titchmarsh [12] in the case $\alpha=1 / 2 ; 3.1$ is the generalization to complex $\alpha$ of the statement (2.47) found in [12, p. 332]. We omit the verification of the fact that every step in Titchmarsh's proof of (2.47) can be directly extended, and quote his assertion [12, p. 323] that "the theory (of $T_{\alpha}$ ) presents no features which do not occur in the case $\alpha=1 / 2$."

THEOREM I. For any $p>1$ there exists a nondecreasing function $f$ such that $\left\|T_{\alpha}\right\|_{p} \leqq f(|\operatorname{Im} \alpha|)$ for any complex $\alpha$.

Proof. From 3.1 we have $\left\|T_{\alpha}\right\|_{p} \leqq\left\|T_{\alpha}\right\|_{s}$ for some $s$ in $M$. But by 2.5 , there exists a nondecreasing $f$ such that $\left\|T_{\alpha}\right\|_{s} \leqq f(|\operatorname{Im} \alpha|)$. The conclusion follows.

Theorem II. If $p>1$, then $T_{\alpha}\left(T_{\lambda} a\right)=T_{\alpha+\lambda} a$ for any $a$ in $S_{p}$.

This is an obvious consequence of 1.3 and Theorem I.

4. Analyticity of $T_{\alpha}$. Let us denote by $\mathscr{E}_{p}$ the set of all bounded linear transformations of $S_{p}$ into itself. We say with Hille [5, p. 53] that a member $V_{\alpha}$ of $\xi_{p}$ is an entire function if $\phi\left(V_{\alpha} x\right)$ is an entire

4 By 1.1(a). 
function of $\alpha$ for every choice of $x$ in $S_{p}$, and for every $\phi$ in the adjoint space $S_{p}^{*}$.

In such a case, there exists $\left[5\right.$, p. 53] a member $V_{\alpha}^{\prime}$ of $\varpi_{p}$ such that

$$
V_{\alpha}^{\prime} x=\lim _{\leftrightarrow \rightarrow 0}\left\{V_{\alpha+\epsilon} x-V_{\alpha} x\right\} \frac{1}{\epsilon} \quad \text { for all } x \text { in } S_{p}
$$

Moreover

$$
\left[V_{\alpha}^{\prime} x\right]_{n}=\frac{d}{d \alpha}\left[V_{\alpha} x\right]_{n} \quad \text { for every } n \text { in } \Omega
$$

We derive (12) from (11) by observing that for any $\phi$ in $S_{p}^{*}$

$$
\phi\left(V_{\alpha}^{\prime} x\right)=\lim _{\epsilon \rightarrow 0}\left\{\phi\left(V_{\alpha+\epsilon} x\right)-\phi\left(V_{\alpha} x\right)\right\} \frac{1}{\epsilon}=\frac{d}{d \alpha} \phi\left(V_{\alpha} x\right) .
$$

Suppose $n \in \Omega$; the above holds in particular for the member $\phi_{n}$ of $S_{p}^{*}$ defined by $\phi_{n}(c)=c_{n}\left(c \in S_{p}\right)$.

THEOREM III. The operator $T_{\alpha}$ is an entire function, and for any $a$ in $S_{p}$ we have

$$
\left[T_{\alpha}^{\prime} a\right]_{n}=\sum_{\nu=-\infty}^{\infty}\left(\frac{d}{d \alpha} t_{\nu}^{\alpha}\right) a_{n-\nu} \quad \text { when } n \in \Omega .
$$

Proof. It is easily seen $[1$, p. 231] that the series representing $\left[T_{\alpha} a\right]_{n}$ is uniformly convergent in any bounded region. Hence $\left[T_{\alpha} a\right]_{n}$ is an entire function of $\alpha$, and the series in (13) represents therefore the derivative of $\left[T_{\alpha} a\right]_{n}$.

From (12) now follows that the theorem will be proved once we have established that $T_{\alpha}$ is an entire function. To that effect, we note that any closed and bounded region $\mathcal{D}$ can be included in a suitable square $|\operatorname{Im} \alpha|<\tau,|\mathrm{Rl} \alpha|<\tau$ and therefore, by Theorem I

$$
\left\|T_{\alpha}\right\|_{p} \leqq f(|\operatorname{Im} \alpha|) \leqq f(\tau) \quad \text { for all } \alpha \text { in } \mathcal{D} \text {. }
$$

From the analyticity of $\left[T_{\alpha} a\right]_{n}=\psi_{\alpha}^{(n)}(a)$ follows that the members $\psi_{\alpha}^{(n)}$ of $S_{p}^{*}$ are analytic. We now refer to [11] for the fact that this allows us to deduce from (14) the analyticity of $T_{\alpha}$ in the arbitrary region $\mathscr{D}$. This completes the proof.

5. The generator. Since $\xi_{p}$ forms a Banach space with norm $\|\cdot\|_{p}$, we can define $\exp \alpha G$ for any $G$ in $\xi_{p}$. The continuity of $T_{\alpha}$ is readily inferred [5, p. 53] from Theorem III. Since further $T_{0} a=a$ and $T_{\alpha} T_{\lambda}=T_{\alpha+\lambda}$, we can conclude from [9] that, when $\alpha$ is real 


$$
T_{\alpha}=\exp \alpha G, \quad \text { where } G=T_{0}^{\prime} .
$$

Both $T_{\alpha}$ and $\exp \alpha G$ being entire functions, we can extend the validity of (15) to all complex values of $\alpha$ by analytic continuation [5, p. 58]. From (15) we see that $G \in \mathbb{E}_{p} ; G$ is the infinitesimal generator of the analytical group $\left\{T_{\alpha} \mid \alpha\right\}$.

The definition of $t^{\alpha}$ readily yields ${ }^{5}$

$$
\left[\frac{d}{d \alpha} t_{n}^{\alpha}\right]_{\alpha=0}=d_{n} \quad(n \in \Omega) .
$$

It now follows from (15) and?(13) that

$$
[G a]_{n}=\sum_{\nu-\infty}^{\infty} d_{\nu} a_{n \rightarrow \nu}=[d * a]_{n} \quad\left(a \in S_{p}, n \in \Omega\right) .
$$

Hence $G$ is identical to the operator defined by (2); this fulfills the aims set forth in the introduction.

\section{BIBLIOGRAPHY}

1. W. L. Ferrar, On the consistency of cardinal function interpolation, Proc. Roy. Soc. Edinburgh vol. 47 (1927) pp. 230-242.

2. H. Hadwiger, Der Begriff der Ultrafunktion, Vierteljahrsschrift der Naturforsch. Gesellschaft Zürich vol. 92 (1947) pp. 31-42.

3. G. H. Hardy, J. E. Littlewood, and G. P6lya, Inequalities, Cambridge, 1934, pp. 212-214 and 225-227.

4. E. Hille, Mathematical Reviews vol. 8 (1947) p. 569.

5. - Functional analysis and semi-groups, Amer. Math. Soc. Colloquium Publications, vol. 31, 1948.

6. E. R. Lorch, The integral representation of weakly almost periodic operators in reflexive vector spaces, Trans. Amer. Math. Soc. vol. 49 (1941) pp. 18-38.

7. W. Magnus, Über einige beschränkten Matrizen, Arch. Math. vol. 2 (1951) pp. 405-412.

8. - On the spectrum of Hilbert's matrix, Amer. J. Math. vol. 72 (1950) pp. 699-704.

9. M. Nagumo, Einige analytische Untersuchungen in linearen metrischen Ringen, Jap. J. Math. vol. 13 (1936) p. 72.

10. M. Riesz, Sur les fonctions conjuguees, Math. Zeit. vol. 27 (1927) pp. 241-243.

11. A. E. Taylor, Linear operations which depend analytically on a parameter, Ann. of Math. (2) vol. 39 (1938) p. 585, $\$ 4$.

12. E. C. Titchmarsh, Reciprocal formulae involving series and integrals, Math. Zeit. vol. 25 (1926) pp. 321-347.

13. - Proc. London Math. Soc. (2) vol. 22 No. 5 iii (1924), also vol. 26 (1927) pp. 1-11, and J. London Math. Soc. vol. 3 (1928) pp. 81-83.

14. J. D. Weston, On the bounds of a bilinear form related to Hilbert's, Quart. J. Math. Oxford Ser. (2) vol. 3 (1952) p. 117.

University of California, Berkeley

b See $\$ 2$ for the definition of $d$. 